\section{REVISTA INTERNACIONAL DE CIENCIAS DEL DEPORTE International Journal of Sport Science}

Rev. int. cienc. deporte

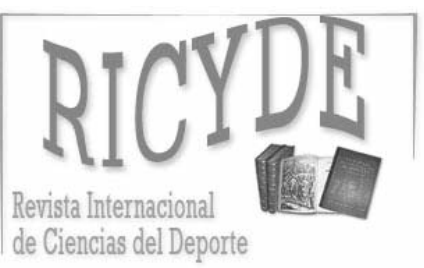

International Journal of Sport Science Volumen VI - Año VI

Páginas:128-140 ISSN:1885-3137 No 19 - Abril - 2010

\title{
Análisis del golpeo de balón y su relación con el salto vertical en futbolistas juveniles de alto nivel. \\ Analysis of the soccer kick and its relationship with the vertical jump in young top-class soccer players.
}

\author{
Daniel Juárez Santos-García \\ Universidad Politécnica de Madrid \\ Cristina López de Subijana \\ Universidad de Alcalá \\ Javier Mallo Sainz \\ Enrique Navarro Cabello \\ Universidad Politécnica de Madrid
}

\section{Resumen}

El rendimiento en acciones explosivas como el golpeo de balón y el salto vertical es de gran relevancia en el fútbol. Los objetivos del presente estudio fueron: 1) Estudiar la secuencia del golpeo en futbolistas jóvenes de alto nivel, y, 2) analizar las posibles relaciones existentes entre el golpeo de balón y el salto vertical. 21 jugadores de alto nivel $(16,1 \pm 0,2$ años) llevaron a cabo un test de salto vertical con contramovimiento $(\mathrm{CMJ})$ y un test de golpeo de balón con la máxima potencia. Los datos fueron registrados mediante una plataforma de fuerzas (Dinascan IBV) y un sistema de captura automática del movimiento (Vicon). Con este estudio, se aporta información sobre la cinemática del golpeo en futbolistas juveniles de alto nivel. En función de los resultados obtenidos, se puede afirmar que la secuencia temporal de máximos de velocidad sucede desde el extremo más proximal al más distal, incrementándose la velocidad. La ausencia de relación entre el golpeo y el salto sugiere el trabajo de fuerza explosiva específico para la mejora de cada una de ellas.

Palabras clave: fútbol; golpeo; salto vertical; potencia.

\section{Abstract}

Kicking and vertical jumping performance are very important in soccer. The aims of the present study were: 1) To study the kinematical sequence of the soccer kick in young top-class soccer players, and, 2 ) to analyse the possible relationships among the different parameters related to the soccer kick and the vertical jump in this population. 21 top-class soccer players $(16,1 \pm 0,2 \mathrm{yr}$.) performed a countermovement jump test (CMJ) and a maximum power soccer kick test. Data was obtained from a Dinascan IBV force platform and the Vicon system of automatic capture of the movement. With this study, we added data on the kinematics of soccer kick in young top-class soccer players. With the results obtained, it is possible to deduce that the temporal sequence of maximum velocities begins from the more proximal to more distal joint, increases the velocity. The absence of relation between these actions suggests the need of a specific explosive strength training for the improvement of each one of them.

Key words: soccer; kicking; vertical jump; power. 
Juárez, D.; López de Subijana, C.; Mallo, J.; Navarro, E. (2010). Análisis del golpeo de balón y su relación con el salto vertical en futbolistas juveniles de alto nivel. Revista Internacional de Ciencias del Deporte. 19(6), 128140. http://www.cafyd.com/REVISTA/01903.pdf

\section{Introducción}

$\mathrm{E}^{n}$ n el fútbol, las acciones decisivas del juego implican la realización de gestos explosivos, por lo que uno de los objetivos del entrenamiento debe ser el aumento del rendimiento en estas acciones. Para ello, resulta fundamental conocer el patrón de ejecución de las mismas. Una de estas acciones es el golpeo de balón.

La cinemática del golpeo de balón en fútbol ha sido ampliamente estudiada (Browder, Tant, y Wilkerson, 1991; Kellis y Katis, 2007; Lees, 1996; Lees, Kershaw, y Moura, 2003; Lees y Nolan, 1998; Lees y Nolan, 2002; Levanon y Dapena, 1998; Parassas, Terauds, y Nathan, 1990; Shan y Westerhoff, 2005; Williams y Griffiths, 2002). Esta acción está caracterizada por una aproximación hacia el balón de uno o más pasos, situando el pie de apoyo al lado del balón y ligeramente por detrás. A continuación, la pierna de golpeo se lleva hacia atrás flexionando la rodilla y, consecutivamente, se inicia el movimiento hacia delante rotando la pelvis sobre la pierna de soporte, llevando el muslo hacia delante mientras la rodilla continúa flexionándose (pre-estiramiento del cuádriceps) hasta que se inicia la extensión. Posteriormente, se produce una deceleración del muslo y una fuerte extensión de la rodilla en el momento en el que el pie impacta con el balón (Kellis y Katis, 2007; Lees y Nolan, 1998).

Con futbolistas senior (19.9 \pm 0.4 años) se llevó a cabo una investigación en la que se examinaron los efectos de un programa de entrenamiento en la cinemática del golpeo (Manolopoulos, Papadopoulos y Kellis, 2006). En este estudio se analizaron, entre otras variables, la velocidad de la cadera, de la rodilla y del tobillo en tres instantes diferentes de la secuencia de golpeo. Sin embargo, en lo que nosotros conocemos, hay muy poca información sobre la cinemática del golpeo en futbolistas juveniles (16-18 años), por lo que existen pocos datos para analizar el posible efecto de la edad en esta acción (Kellis y Katis, 2007). En algunos estudios, se ha llegado a la conclusión de que variables como la velocidad del balón en el golpeo y la velocidad angular de la rodilla incrementan con la edad (Capranica et al., 1992; Luhtanen, 1988). En el estudio realizado por Luhtanen (1988), con jugadores de entre 10 y 17 años, se registraron valores de velocidad de balón de entre 14.9 y $22.2 \mathrm{~m} / \mathrm{s}$ en golpeos de empeine con dos pasos de aproximación. Por su parte, Rodano y Tavana (1993), con 10 futbolistas profesionales jóvenes (17.6 \pm 0.5 años) encontraron velocidades del balón en el golpeo de empeine de entre 22.3 y $30 \mathrm{~m} / \mathrm{s}$. El incremento en la velocidad del balón puede ser debido al incremento de la masa muscular de los sujetos y a mejoras de la técnica de golpeo (Poulmedis et al., 1988; Rodano y Tavana, 1993; Taïana et al., 1993; Trolle et al., 1993). En cualquier caso, ya en edades jóvenes se han llegado a encontrar valores de velocidad del balón en el golpeo de empeine de más de $32 \mathrm{~m} / \mathrm{s}$ en futbolistas con alto nivel de destreza en esta acción (Nunome et al., 2006a), valores éstos superiores a los observados en otros estudios con jugadores de gran experiencia y de mayor edad (Dorge et al., 2002; Nunome et al., 2002; Nunome et al., 2006b). En el citado estudio de Rodano y Tavana (1993), además de registrar la velocidad del balón en el golpeo de empeine en futbolistas jóvenes, se analizó la velocidad en el momento del impacto con el balón en 5 marcadores colocados en los sujetos: cresta ilíaca, cadera, rodilla, tobillo y quinto metatarsiano. En esta investigación, no se encontraron buenas correlaciones entre la velocidad de dichos marcadores. Los valores de velocidad fueron relativamente bajos, por lo que parece que otros aspectos, como la rigidez de la pierna en el impacto y la posición relativa entre el pie y el balón influyen en gran medida en la velocidad del balón. Se encontró que las correlaciones de la velocidad de los marcadores establecidos en el momento del impacto con el balón y la fuerza de reacción en el suelo, con 
Juárez, D.; López de Subijana, C.; Mallo, J.; Navarro, E. (2010). Análisis del golpeo de balón y su relación con el salto vertical en futbolistas juveniles de alto nivel. Revista Internacional de Ciencias del Deporte. 19(6), 128140. http://www.cafyd.com/REVISTA/01903.pdf

respecto a la velocidad del balón, son estrictamente dependientes del sujeto considerado, por lo que no se pueden establecer criterios generales.

Por otro lado, de cara a una óptima planificación y entrenamiento específico de las distintas acciones explosivas realizadas en el fútbol, resultará interesante conocer la relación existente entre el rendimiento en estas acciones. Los estudios en el fútbol sobre la posible relación de la velocidad máxima de tiro con otros tests de carácter explosivo parecen escasos. En un estudio con futbolistas (Cabri, De Proft, Dufour, y Clarys, 1988) se observó que la correlación entre la distancia alcanzada por el balón en un golpeo máximo en fútbol y la altura de salto vertical estirando la mano era significativamente elevada $(0.69 ; \rho<0.05)$. Por otro lado, en un estudio con 55 futbolistas infantiles y cadetes (Mercé, González, Mayo, Pardo, y Sorli, 2004), se determinó la correlación entre la velocidad media de golpeos con la máxima potencia y otros tests de carácter explosivo, obteniendo coeficientes de correlación significativos y medios-bajos (en torno a 0.40 en acciones de salto y aceleración en carrera). En un estudio llevado a cabo con jugadores de fútbol sala de alto nivel no se encontraron relaciones entre la velocidad del balón en golpeos con el empeine con la máxima potencia y la altura de salto sin contramovimiento (Squat Jump-SJ), con contramovimiento (Countermovement Jump- CMJ) y el tiempo en 5, 10 y 20 m de carrera a la máxima velocidad (Juárez y Navarro, 2006a).

Por ello, los objetivos que se plantearon con la presente investigación fueron: 1) Estudiar la secuencia cinemática del golpeo en futbolistas jóvenes de alto nivel, y 2) analizar las posibles relaciones existentes entre el rendimiento en el golpeo de balón y el salto vertical.

\section{Material y método}

\section{Muestra}

Para la realización de este estudio se contó con la participación de 21 futbolistas juveniles de alto nivel (16.1 \pm 0.2 años; $1.77 \pm 0.06 \mathrm{~m} ; 67.7 \pm 6.3 \mathrm{~kg})$. Estos jugadores pertenecían a un equipo de las categorías inferiores de un club de la Primera División Española, seleccionados entre una amplia muestra para formar parte de ese equipo. Algunos de ellos han sido internacionales en categorías inferiores, y entrenan un mínimo de 4 días por semana con un partido de competición semanal. Los padres de los participantes autorizaron por escrito la participación de sus hijos en el estudio, a la vez que los participantes dieron su consentimiento por escrito.

\section{Procedimiento}

Los tests fueron realizados en el Laboratorio de Biomecánica Deportiva de la Facultad de Ciencias de la Actividad Física y del Deporte de la Universidad Politécnica de Madrid.

Antes de la aplicación de los tests los participantes llevaron a cabo un calentamiento estandarizado de 15 min de duración que consistía en lo siguiente: 5 minutos de carrera suave; estiramientos: psoas, cuádriceps, aductores, gemelos. 2 × 8 segundos con cada pierna; 8 saltos verticales con contramovimiento (Countermovement Jump-CMJ) submáximos, y 8 golpeos submáximos.

- Salto vertical con contramovimiento (CMJ): el sujeto se colocaba de pie, con los pies separados a la anchura de los hombros, y situando cada pie en una plataforma de fuerzas diferente incrustadas en el suelo (la pierna dominante de golpeo se situaba en la que se denominó plataforma 1 y la otra en la plataforma 2), con las manos en las caderas y las rodillas extendidas. La ejecución consistía en un salto vertical con movimiento de flexión 
Juárez, D.; López de Subijana, C.; Mallo, J.; Navarro, E. (2010). Análisis del golpeo de balón y su relación con el salto vertical en futbolistas juveniles de alto nivel. Revista Internacional de Ciencias del Deporte. 19(6), 128140. http://www.cafyd.com/REVISTA/01903.pdf

previo de piernas (Bosco, 1994), sin flexionar la espalda ni levantar los talones del suelo. Se realizaban 3 ensayos con 20 segundos de descanso entre ellos. Para el análisis posterior, se seleccionaba el salto en el que se conseguía la mayor altura.

- Test de golpeo de balón: el balón estaba situado en el suelo, junto a una de las plataformas de fuerzas. En función de la pierna de golpeo, el balón se colocaba a un lado u otro de la plataforma para que el participante situara su pie de apoyo en el momento del golpeo sobre la plataforma. El sujeto se aproximaba al balón con una carrera de 5 metros, con la velocidad que creyera conveniente para golpear el balón con el empeine con la máxima potencia hacia una red situada 5 metros por delante del balón (Markovic, Dizdar, y Jaric, 2006). No se rechazó ningún golpeo por el lugar de impacto del balón en la red. Los 3 intentos de cada participante se realizaban con un intervalo de descanso de $30 \mathrm{~s}$ (Markovic, Dizdar, y Jaric, 2006). Para el análisis posterior, se seleccionaba el golpeo en el que se conseguía la mayor velocidad de salida del balón.

Para los golpeos se utilizó un balón aprobado por la FIFA (Fédération Internationale de Football Association) con una presión de inflado estandarizada.

Los datos referidos a los saltos fueron registrados mediante dos plataformas dinamométricas Dinascan del Instituto de Biomecánica de Valencia (IBV) con una frecuencia de 250 Herzios $(\mathrm{Hz})$, lo cual parece suficiente a tenor de otros estudios en los que también se han empleado plataformas de fuerzas para registrar saltos verticales, y en los que se han empleado incluso frecuencias de muestreo inferiores (Driss et al., 2001). Los datos se filtraron con un filtro de paso bajo a $50 \mathrm{~Hz}$ y posteriormente se exportaron a ficheros de texto. También se utilizó una de estas plataformas para registrar las fuerzas de reacción de la pierna de apoyo en el golpeo del balón.

Los golpeos fueron grabados mediante un sistema de captura del movimiento en tres dimensiones (VICON Motion Systems, Oxford Metrics Ltd., Oxford, England), utilizando 6 cámaras a $250 \mathrm{~Hz}$. Antes del calentamiento, se fijaron 31 marcadores reflectantes de 14 milímetros de diámetro sobre el cuerpo de los participantes y dos sobre el balón, diametralmente opuestos (Kellis, Katis, y Vrabas, 2006). Estos marcadores permitían una libertad de movimientos considerable, por lo que no influyeron negativamente en el rendimiento de las acciones llevadas a cabo. Posteriormente, mediante un software específico, los datos fueron filtrados usando funciones Spline de quinto orden según el método de ajuste Woltring-CGV (Cross General Validation), y se calcularon las variables del estudio. La fuerza ejercida por la pierna de apoyo durante los golpeos fue registrada mediante una plataforma dinamométrica Dinascán IBV.

\section{Variables}

Las variables que se analizaron fueron las siguientes:

CMJ:

o Altura de salto (centímetros- $\mathrm{cm}$ ): se obtiene en función de la velocidad de despegue, integrando los datos registrados en dos plataformas, ya que el salto se realizaba con un pie en cada una de ellas.

o Potencia máxima total (watios -w)

o Potencia máxima relativa a la masa corporal (watios/kilogramo- $\mathrm{w} / \mathrm{kg}$ )

o Fuerza Vertical máxima total (Newton- N).

o Fuerza Vertical máxima relativa al peso corporal (veces el peso corporal- BW). 
Juárez, D.; López de Subijana, C.; Mallo, J.; Navarro, E. (2010). Análisis del golpeo de balón y su relación con el salto vertical en futbolistas juveniles de alto nivel. Revista Internacional de Ciencias del Deporte. 19(6), 128140. http://www.cafyd.com/REVISTA/01903.pdf

o Fuerza Vertical máxima aplicada en cada apoyo, en la plataforma 1 y en la plataforma $2(\mathrm{~N})$. La pierna que se situaba en la plataforma 1 era la pierna de golpeo preferente.

Todas estas variables fueron seleccionadas del ensayo en el que se consiguió mayor altura de salto.

Golpeo de balón:

o Velocidad máxima (metros/segundo- $\mathrm{m} / \mathrm{s}$ ) del balón: el valor más alto de velocidad del punto medio de los dos marcadores situados diametralmente opuestos en el balón

o Velocidad lineal máxima $(\mathrm{m} / \mathrm{s})$ del pie de golpeo.

o Velocidad lineal máxima $(\mathrm{m} / \mathrm{s})$ del tobillo de la pierna de golpeo.

o Velocidad lineal máxima $(\mathrm{m} / \mathrm{s})$ de la rodilla de la pierna de golpeo.

o Velocidad lineal máxima $(\mathrm{m} / \mathrm{s})$ de la cadera de la pierna de golpeo.

o Fuerza vertical máxima absoluta $(\mathrm{N})$ en la pierna de apoyo.

o Fuerza vertical máxima relativa al peso corporal (BW) en la pierna de apoyo.

o Tiempo (segundos- s) en el que ocurre la velocidad máxima de rodilla (considerando como tiempo $0-\mathrm{t}_{0^{-}}$el instante el que tiene lugar el máximo de velocidad de la cadera) $\left(\mathrm{t}_{1}\right)$.

o Tiempo (s) en el que ocurre la velocidad máxima de tobillo $\left(t_{2}\right)$.

o Tiempo (s) en el que ocurre la velocidad máxima del pie $\left(\mathrm{t}_{3}\right)$.

o Fase 1 (s): tiempo que transcurre desde $t_{0}$ a $t_{1}$.

o Fase 2 (s): tiempo que transcurre desde $t_{1}$ a $t_{2}$.

o Fase 3 (s): tiempo que transcurre desde $t_{2}$ a $t_{3}$.

Todas estas variables fueron seleccionadas del ensayo en el que se consiguió mayor velocidad máxima del balón.

\section{Análisis estadístico}

Para el análisis estadístico se utilizó el programa SPSS v.16.0 (SPSS Inc., Chicago, IL). Se comprobó la normalidad de la muestra mediante la prueba de Kolmogorov-Smirnov, y la fiabilidad de cada una de las variables analizadas (considerando los 3 ensayos realizados tanto en el CMJ como en el golpeo) mediante el Coeficiente de Correlación Intraclase (CCI) y el Coeficiente de Variación (CV). Se calculó la media y la desviación estándar (Media \pm DS) de cada una de las variables de estudio. Para analizar las diferencias entre cada una de las velocidades máximas de los marcadores articulares de los ensayos en los que se conseguía la mayor velocidad máxima del balón se aplicó un ANOVA de medidas repetidas. También se aplicó un ANOVA de medidas repetidas para estudiar las diferencias entre los tiempos en los que se producen los máximos de velocidad y entre la duración de las fases. Para analizar las relaciones entre las variables analizadas en el golpeo de balón y las analizadas en el salto vertical se aplicó la prueba de correlación de Pearson. El nivel de significación se fijó en $\mathrm{p}<0.05$. 
Juárez, D.; López de Subijana, C.; Mallo, J.; Navarro, E. (2010). Análisis del golpeo de balón y su relación con el salto vertical en futbolistas juveniles de alto nivel. Revista Internacional de Ciencias del Deporte. 19(6), 128140. http://www.cafyd.com/REVISTA/01903.pdf

\section{Resultados}

Todas las variables analizadas en el presente estudio mostraron un CCI mayor de $0.8(\mathrm{p}<0.01)$ y un CV menor del 6\%.

En la tabla 1 se muestran los datos obtenidos en las variables de velocidad y fuerza en el golpeo de balón. Se encontraron diferencias $(\mathrm{p}<0.01)$ entre las velocidad máximas de cada marcador articular, siendo la más alta la del pie, seguida por tobillo, rodilla y cadera. La secuencia de velocidades puede observarse en la figura 1.

Los datos referentes a las variables temporales analizadas en el golpeo de balón son ofrecidos en la tabla 2. Se encontraron diferencias $(\mathrm{p}<0.01)$ entre los tiempos en los que se producen los máximos de velocidad de cada marcador articular, excepto entre el tiempo en el que se produce el máximo de velocidad del tobillo $\left(\mathrm{t}_{2}\right)$ y el del pie $\left(\mathrm{t}_{3}\right)$. También existen diferencias $(\mathrm{p}<0.01)$ entre la duración de las fases, siendo la mayor la fase 1 (tiempo entre el máximo de la cadera y el máximo de la rodilla).

Tabla 1. Variables de velocidad y fuerza analizadas en el golpeo de balón.

\begin{tabular}{|c|c|}
\hline VARIABLES & Media \pm DS \\
\hline $\begin{array}{c}\text { Velocidad máxima } \\
\text { del balón }\end{array}$ & $30.06 \pm 1.54 \mathrm{~m} / \mathrm{s}$ \\
\hline $\begin{array}{c}\text { Velocidad máxima } \\
\text { del pie }\end{array}$ & $24.59 \pm 1.33 \mathrm{~m} / \mathrm{s}^{* *}$ \\
\hline $\begin{array}{c}\text { Velocidad máxima } \\
\text { del tobillo }\end{array}$ & $19.23 \pm 0.88 \mathrm{~m} / \mathrm{s}^{* *}$ \\
\hline $\begin{array}{c}\text { Velocidad máxima } \\
\text { de la rodilla }\end{array}$ & $11.04 \pm 0.58 \mathrm{~m} / \mathrm{s}^{* *}$ \\
\hline $\begin{array}{c}\text { Velocidad máxima } \\
\text { de la cadera }\end{array}$ & $5.48 \pm 0.58 \mathrm{~m} / \mathrm{s}^{* *}$ \\
\hline $\begin{array}{c}\text { Fuerza vertical } \\
\text { máxima }\end{array}$ & $2496.29 \pm 690.51 \mathrm{~N}$ \\
\hline $\begin{array}{c}\text { Fuerza vertical } \\
\text { máxima relativa }\end{array}$ & $3.71 \pm 0.85 \mathrm{BW}$ \\
\hline
\end{tabular}

$* *(p<0.01$ con el resto de velocidades de puntos articulares $)$ 
Juárez, D.; López de Subijana, C.; Mallo, J.; Navarro, E. (2010). Análisis del golpeo de balón y su relación con el salto vertical en futbolistas juveniles de alto nivel. Revista Internacional de Ciencias del Deporte. 19(6), 128140. http://www.cafyd.com/REVISTA/01903.pdf

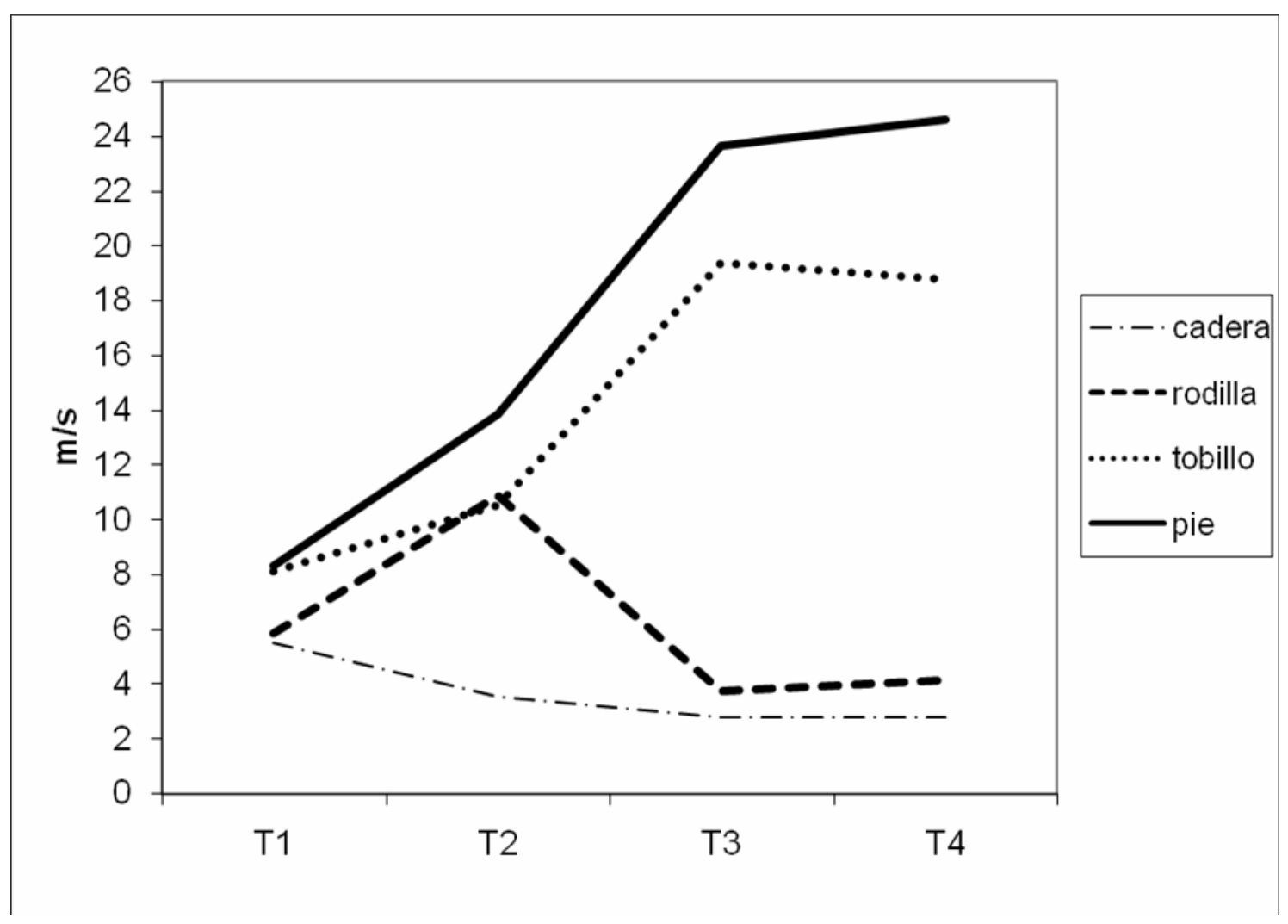

Figura 1.- Secuencia de velocidades en el golpeo de balón.

Tabla 2. Variables temporales analizadas en el golpeo de balón.

\begin{tabular}{|c|c|}
\hline VARIABLES & Media \pm DS \\
\hline$t_{0}$ & $0 \mathrm{~s}^{* *}$ \\
\hline$t_{1}$ & $0.159 \pm 0.021 \mathrm{~s}^{\Delta \Delta}$ \\
\hline $\mathrm{t}_{2}$ & $0.163 \pm 0.021 \mathrm{~s}^{\Delta \Delta}$ \\
\hline $\mathrm{t}_{3}$ & $0.103 \mathrm{~s}$ \\
\hline Fase 1 & $0.056 \mathrm{~s}$ \\
\hline Fase 2 & $0.004 \mathrm{~s}$ \\
\hline Fase 3 & \\
\hline
\end{tabular}

**( $(\mathrm{p}<0.01$ con el resto de tiempos en los que se producen los máximos de velocidad $)$

${ }^{\Delta \Delta}\left(\mathrm{p}<0.01\right.$ con $\mathrm{t}_{0} \mathrm{y}$ con $\left.\mathrm{t}_{1}\right)$.

$(\mathrm{p}<0.01$ con la duración del resto de fases) 
Juárez, D.; López de Subijana, C.; Mallo, J.; Navarro, E. (2010). Análisis del golpeo de balón y su relación con el salto vertical en futbolistas juveniles de alto nivel. Revista Internacional de Ciencias del Deporte. 19(6), 128140. http://www.cafyd.com/REVISTA/01903.pdf

En la tabla 3 se muestran los datos obtenidos en las variables analizadas en el CMJ, tanto la altura de salto conseguida, como la fuerza máxima aplicada por ambas piernas y de forma individualizada por cada pierna, y la potencia máxima total.

Table 3. Variables analizadas en el CMJ

\begin{tabular}{|c|c|}
\hline VARIABLES & Media \pm DS \\
\hline Altura de salto & $0.41 \pm 0.08 \mathrm{~m}$ \\
\hline $\begin{array}{c}\text { Potencia } \\
\text { máxima }\end{array}$ & $3658.30 \pm 556.81 \mathrm{~W}$ \\
\hline $\begin{array}{c}\text { Potencia } \\
\text { máxima relativa }\end{array}$ & $54.67 \pm 6.69 \mathrm{~W} / \mathrm{kg}$ \\
\hline $\begin{array}{c}\text { Fuerza vertical } \\
\text { máxima }\end{array}$ & $1669.84 \pm 166.54 \mathrm{~N}$ \\
\hline $\begin{array}{c}\text { Fuerza vertical } \\
\text { máxima relativa }\end{array}$ & $2.54 \pm 0.27 \mathrm{BW}$ \\
\hline $\begin{array}{c}\text { Fuerza vertical } \\
\text { máxima en } \\
\text { plataforma } 1\end{array}$ & $860.25 \pm 98.96 \mathrm{~N}$ \\
\hline $\begin{array}{c}\text { Fuerza vertical } \\
\text { máxima en } \\
\text { plataforma } 2\end{array}$ & $809.59 \pm 92.88 \mathrm{~N}$ \\
\hline
\end{tabular}

Al analizar los coeficientes de correlación obtenidos entre las principales variables de rendimiento de ambas acciones, es decir, la velocidad de salida del balón y la altura de salto, se observó un coeficiente de correlación bajo (-0.09) y no significativo.

Los tiempos en los que se producen los máximos de velocidad de los marcadores articulares no presentaron relación con ninguna de las variables de salto analizadas.

\section{Discusión}

Al analizar la secuencia de máximos de velocidad se comprobó que, como ocurre en general en movimientos rápidos de cadena abierta, los segmentos proximales alcanzan antes su máximo de velocidad que los distales (Putnam, 1991). Una importante característica de la secuencia de movimientos proximal-distal es el decrecimiento de la velocidad angular del segmento proximal mientras el distal incrementa su valor máximo (Putnam, 1991).

La secuencia temporal en la que se producen los máximos de velocidad de los centros articulares de la cadena cinética abierta dependen del tipo de destreza, pudiendo ser indicativos del rendimiento técnico (López de Subijana, de Antonio, Juárez y Navarro, 2008; Navarro, Cabrero y Vizcaíno, 1998). En nuestro caso, se ha observado que el tiempo desde el inicio de la fase de golpeo (máximo de velocidad del marcador de la cadera) hasta el instante en el que se alcanza el máximo de velocidad de la rodilla representa el 64\% de la fase.

En cuanto a la velocidad máxima del balón, la media observada en nuestro estudio (aproximadamente $30 \mathrm{~m} / \mathrm{s}$ ) es similar a la observada en otro estudio con futbolistas jóvenes, (Helgerud, Engen, Wisloff, y Hoff, 2001), y muy superior a la encontrada en otro trabajo 
Juárez, D.; López de Subijana, C.; Mallo, J.; Navarro, E. (2010). Análisis del golpeo de balón y su relación con el salto vertical en futbolistas juveniles de alto nivel. Revista Internacional de Ciencias del Deporte. 19(6), 128140. http://www.cafyd.com/REVISTA/01903.pdf

$(23.6 \pm 2.5 \mathrm{~m} / \mathrm{s})$ también con futbolistas jóvenes (Mognoni, Narici, Sirtori, y Lorenzelli, 1994). Este hecho puede estar relacionado con la metodología empleada para la realización del golpeo de balón en esta última investigación. En el estudio de Mognoni et al. (1994), el test implicaba, a diferencia de nuestro trabajo, una precisión en el golpeo, lo que produce una disminución en la velocidad del balón (Juárez y Navarro, 2006b). También en el citado estudio, lo que se midió fue la velocidad media de la trayectoria del balón, a diferencia del nuestro en el que se midió la velocidad máxima del balón. De igual forma, la carrera de aproximación fue más reducida en ese trabajo que en el nuestro, lo que puede también contribuir a las diferencias observadas (Isokawa y Lees, 1988; Kellis y Katis, 2007; Opavsky, 1988).

La velocidad máxima del pie $(24.59 \pm 1.33 \mathrm{~m} / \mathrm{s})$ en la acción del golpeo fue superior a la determinada en otros estudios llevados a cabo con futbolistas de gran experiencia, en los que se encontraron valores de $21.50 \pm 2.21 \mathrm{~m} / \mathrm{s}$ (Barfield, 1995) y $20.4 \pm 1.3 \mathrm{~m} / \mathrm{s}$ (Barfield, Kirkendall, y Yu, 2002). Cabe resaltar que en dichos estudios la velocidad máxima del balón fue también inferior a la encontrada en nuestro trabajo, siendo $26.4 \pm 2.09 \mathrm{~m} / \mathrm{s}$ en el estudio de Barfield (1995), y $25.3 \pm 1.51 \mathrm{~m} / \mathrm{s}$ en el de Barfield et al. (2002). En ambos estudios la carrera de aproximación para golpear el balón ( 2 pasos) fue más limitada que en nuestra investigación $(5 \mathrm{~m})$, lo que puede ser una de las causas de las diferencias en velocidad encontradas. La velocidad máxima registrada en el marcador de la rodilla también fue superior en nuestro estudio (11.04 $\pm 0.58 \mathrm{~m} / \mathrm{s})$ a la encontrada por Barfield (1995) en 18 futbolistas con experiencia $(10.05 \pm 0.92 \mathrm{~m} / \mathrm{s})$, posiblemente también afectado por las diferencias en la velocidad de la carrera de aproximación.

En los golpeos de balón realizados, se encontró una fuerza vertical máxima en la pierna de apoyo en el momento del golpeo de $2694.44 \pm 616.59 \mathrm{~N}$, lo que supone la aplicación de una fuerza de aproximadamente 3.7 veces el peso corporal del sujeto, superior a la fuerza relativa encontrada en otro estudio (3.2 veces el peso corporal) con futbolistas de alto nivel y edad similar a los que participaron en nuestro trabajo (Rodano y Tavana, 1993). Estas diferencias pueden ser debidas a las características de realización del test de golpeo, ya que en el citado estudio de Rodano y Tavana (1993), los futbolistas realizaban el golpeo de balón con tan sólo dos pasos de carrera.

En cuanto a la altura media de salto encontrada en el presente trabajo fue superior a la hallada en 21 futbolistas jóvenes de élite (Venturelli, Trentin, y Bucci, 2007), y similar a la obtenida en un estudio con futbolistas profesionales (Cometti, Maffiuletti, Pousson, Chatard, y Maffulli, 2001). Esto puede ser debido a que un salto con contramovimiento con las manos en las caderas no es una acción específica del fútbol, por lo que sujetos de edad o niveles inferiores pueden presentar valores similares o superiores a futbolistas de mayor nivel. En cualquier caso, hay que ser cauto a la hora de comparar resultados de diversos estudios, ya que aspectos como la instrumentación utilizada o el momento de realización de los tests pueden influir en gran medida en los resultados.

La fuerza vertical máxima aplicada en el CMJ fue equivalente a 2 veces y media el peso corporal del sujeto, valor similar al encontrado en 161 aspirantes al ingreso en una Facultad de Ciencias del Deporte (Lara, Abián, Alegre, Jiménez, y Aguado, 2005). Los datos de fuerza aplicada por ambas piernas, recogidos en situación normal pueden ser especialmente útiles para el control del proceso de recuperación de jugadores lesionados, observando la diferencia entre la fuerza ejercida por ambas piernas durante dicho proceso y su aproximación a los valores habituales del sujeto. 
Juárez, D.; López de Subijana, C.; Mallo, J.; Navarro, E. (2010). Análisis del golpeo de balón y su relación con el salto vertical en futbolistas juveniles de alto nivel. Revista Internacional de Ciencias del Deporte. 19(6), 128140. http://www.cafyd.com/REVISTA/01903.pdf

El hecho de haber encontrado un coeficiente de correlación bajo y no significativo entre las principales variables de rendimiento del golpeo de balón y del salto vertical, es decir, entre la velocidad de salida del balón en el golpeo, y la altura de salto vertical, denota, en principio, que en los futbolistas que participaron en este estudio no existe relación entre el rendimiento obtenido en ambas acciones. Este hecho puede ser debido a factores como la participación muscular en cada una de estas acciones, y a la técnica y coordinación de cada sujeto al realizarlas. En un estudio llevado a cabo con 10 jugadores de fútbol sala de División de Plata (24.7 \pm 3.0 años), tampoco se encontró relación entre la altura de salto en CMJ y la velocidad del balón en golpeos con la máxima potencia (Juárez y Navarro, 2006a). Esto sugiere la idea del entrenamiento específico de cada una de estas acciones para intentar optimizar el rendimiento de las mismas.

En cualquier caso, también debe considerarse que ni los golpeos ni los saltos se realizaron en situaciones reales de juego. Los saltos realizados habitualmente en fútbol presentan diferencias con los llevados a cabo en este estudio, ya que en el juego se salta en muchas ocasiones batiendo con una sola pierna, que además suele no coincidir con la pierna dominante del golpeo.

Por su parte, otros autores (Cabri et al., 1988) han obtenido, en una muestra formada por 11 futbolistas de alto nivel con una edad media de 20 años, un coeficiente de correlación alto $(0.69, \rho<0.05)$ entre el golpeo y el salto, pero hay que tener en cuenta que en dicho estudio, lo que se correlacionó fue la distancia alcanzada con el golpeo y la altura de salto vertical tocando con la mano una pared al elevarse. También en otro estudio (Mercé et al., 2004) se encontró una relación significativa entre la velocidad media del balón en golpeos con la máxima potencia y saltos sin y con contramovimiento, pero en este caso habría que considerar que la muestra estaba formada por 55 futbolistas infantiles y cadetes $(14.59 \pm 1.08$ años). En cualquier caso, los coeficientes encontrados fueron de valor medio-bajo.

\section{Conclusiones}

En el presente estudio se ha aportado información relativa a la acción de golpeo de balón y el salto vertical en jóvenes futbolistas. Como se esperaba, la secuencia temporal de máximos de velocidad en la acción de golpeo sucede desde el extremo más proximal al más distal, incrementándose la velocidad.

La ausencia de relación entre el golpeo y el salto sugiere el trabajo de fuerza explosiva específico para la mejora de cada una de ellas. 
Juárez, D.; López de Subijana, C.; Mallo, J.; Navarro, E. (2010). Análisis del golpeo de balón y su relación con el salto vertical en futbolistas juveniles de alto nivel. Revista Internacional de Ciencias del Deporte. 19(6), 128140. http://www.cafyd.com/REVISTA/01903.pdf

\section{Referencias bibliográficas}

Barfield, W. (1995). Effects of selected kinematic and kinetic variables on instep kicking with dominant and non-dominant limbs. Journal of Human Movement Studies, 29(6), 251-272.

Barfield, W.; Kirkendall, D. T., \& Yu, B. (2002). Kinematic instep kicking differences between elite female and male soccer players. Journal of Sports Science and Medicine, 1(3), 72-79.

Bosco, C. (1994). La valoración de la fuerza con el test de Bosco. Barcelona: Paidotribo.

Browder, K. D.; Tant, C. L., \& Wilkerson, J. D. (1991). A three dimensional kinematic analysis of three kicking techniques in female players. En C. L. Tant, P. E. Patterson y S. L. York (Eds.), Biomechanics in Sport IX (pp. 95-100). Ames, IA: ISU Press.

Cabri, J.; De Proft, E.; Dufour, W., \& Clarys, J. P. (1988). The relation between muscular strength and kick performance. En T. Reilly, A. Lees, K. Davids y W. J. Murphy (Eds.), Science and Football (Vol. I, pp. 186-193). London: E \& FN Spon.

Capranica, L.; Cama, G.; Fanton, F.; Tessitore, A., \& Figura, F. (1992). Force and power of preferred and non-preferred leg in young soccer players. Journal of Sports Medicine and Physical Fitness, 32(4), 358-363.

Cometti, G.; Maffiuletti, N. A.; Pousson, M.; Chatard, J. C., \& Maffulli, N. (2001). Isokinetic strength and anaerobic power elite, subelite and amateur french soccer players. International J ournal of Sports Medicine, 22(1), 45-51.

Dorge, H.; Bull-Andersen, T.; Sorensen, H.; \& Simonsen, E. (2002). Biomechanical differences in soccer kicking with the preferred and the non-preferred leg. Journal of Sports Sciences, 20(4), 293-299.

Driss, T.; Vandewalle, H.; Quievre, J.; Millar, C.; Monod, H. (2001). Effects of external loading on power output in a squat jump on a force platform: a comparison between strength and power athletes and sedentary individuals. Journal of Sports Sciences, 19(2), 99-105.

Helgerud, J.; Engen, L. C.; Wisloff, U., \& Hoff, J. (2001). Aerobic endurance training improves soccer performance. Medicine and Science in Sports and Excercise, 33(11), 1925-1931.

Isokawa, M. \& Lees, A. (1988). A biomechanical analysis of the instep kick motion in soccer. En T. Reilly, A. Lees, K. Davids, y W. J. Murphy (Eds.), Science and Football (Vol. I, pp. 449-455). London: E \& FN Spon.

Juárez, D., y Navarro, F. (2006a). Análisis de la velocidad del balón en el golpeo en jugadores de fútbol sala en función del sistema de medición, la intención en la precisión del tiro, y su relación con otras acciones explosivas. Motricidad - European J ournal of Human Movement, XV(especial), 149-157.

Juárez, D., y Navarro, F. (2006b). Análisis de la velocidad del balón en el tiro en futbolistas en función de la intención de precisión. Motricidad - European Journal of Human Movement, XVI, 39-49.

Kellis, E., \& Katis, A. (2007). Biomechanical characteristics and determinants of instep soccer kick. Journal of Sports Science and Medicine, 6(2), 154-165.

Kellis, E.; Katis, A., \& Vrabas, I. S. (2006). Effects of an intermittent exercise fatigue protocol on biomechanics of soccer kick performance. Scandinavian Journal of Medicine and Science in Sports, 16(5), 334-344. 
Juárez, D.; López de Subijana, C.; Mallo, J.; Navarro, E. (2010). Análisis del golpeo de balón y su relación con el salto vertical en futbolistas juveniles de alto nivel. Revista Internacional de Ciencias del Deporte. 19(6), 128140. http://www.cafyd.com/REVISTA/01903.pdf

Lara, A.; Abián, J.; Alegre, L.; Jiménez, L., y Aguado, X. (2005). Tests de salto con plataforma de fuerzas en una población de aspirantes al ingreso en una Facultad de Educación Física. Extraído el 8 de abril de 2005, de htt://www.uclm.es

Lees, A. (1996). Biomechanics applied to soccer skills. En T. Reilly (Ed.), Science and Football (pp. 123-133). London: E \& FN Spon.

Lees, A.; Kershaw, L., \& Moura, F. (2003). The three dimensional nature of the maximal instep kick in soccer. In World Congress on Science and Football - 5. Book of abstracts (pp. 126-127). Madrid: Gymnos.

Lees, A., \& Nolan, L. (1998). The biomechanics of soccer: A review. Journal of Sports Sciences, 16, 211-234.

Lees, A., \& Nolan, L. (2002). Three-dimensional kinematic analysis of the instep kick under speed and accuracy conditions. En W. Spinks, T. Reilly y A. Murphy (Eds.), Science and Football (Vol. IV, pp. 16-21). London: Routledge.

Levanon, J., \& Dapena, J. (1998). Comparison of the kinematics of the full-instep and pass kicks in soccer. Medicine and Science in Sports and Exercise, 30(6), 917-927.

López de Subijana, C.; de Antonio, R.; Juarez, D., y Navarro, E. (2008). El timing en el swing de golf de jóvenes promesas. Kronos, XII, 135-147.

Markovic, G., Dizdar, D., y Jaric, S. (2006). Evaluation of tests of maximum kicking performance. J ournal of Sports Medicine and Physical Fitness, 46(2), 215-220.

Luhtanen, P. (1988). Kinematics and kinetics of maximal instep kicking in junior soccer players. En T. Reilly, A. Lees, K. Davids, y W. J. Murphy. (Eds.), Science and Football (Vol. I, pp. 441-448). London: E \& FN Spon.

Mercé, J.; González, L. M.; Mayo, C.; Pardo, A., y Sorli, J. (2004). Evaluación de la condición física específica en jugadores infantiles y cadetes de fútbol. En G. Brizuela, S. Llana y J. F. Guzmán (Eds.), III Congreso de la Asociación Española de Ciencias del Deporte. Hacia la Convergencia Europea. (Formato CD). Valencia.

Mognoni, P.; Narici, M. V.; Sirtori, M. D., \& Lorenzelli, F. (1994). Isokinetic torques and kicking maximal ball velocity in young soccer players. Journal of Sports Medicine and Physical Fitness, 34(4), 357-361.

Navarro, E.; Cabrero, O., \& Vizcaíno, F. (1998). A three-dimensional analysis of angular velocities of segments in javelin throwing. En H. J. Riehle y M. M. Vieten (Eds.), Proceedings of the 16th ISBS Syposium on Biomechanics on Sport (pp. 204-207). Universidad de Konstanz, Alemania.

Nunome, H.; Asai, T.; Ikegami, Y., \& Sakurai, S. (2002). Three-dimensional kinetic analysis of side-foot and instep soccer kicks. Medicine and Science in Sports And Exercise, 34(12), 2028-2036.

Nunome, H., Ikegami, Y., Lozakai, R., Apriantono, T. y Sano, S. (2006a). Segmental dynamics of soccer instep kickin with the preferred and non-preferred leg. Journal of Sports Sciences, 24(5), 529-541.

Nunome, H.; Lake, M.; Georgakis, A., \& Stergioulas, L. K. (2006b). Impact phase kinematics of instep kicking in soccer. J ournal of sports Sciences, 24(1), 11-22.

Opavsky, P. (1988). An investigation of linear and angular kinematics of the leg during two types of soccer kick. En T. Reilly, A. Lees, K. Davids, y W. J. Murphy (Eds.), Science and Football (Vol. I, pp. 456-459). London: E \& FN Spon.

Parassas, S. G.; Terauds, J., \& Nathan, T. (1990). Three dimensional kinematic analysis of high and low trajectory kicks in soccer. Ponencia presentada en el VIIIth Symposium of the International Society of Biomechanics in Sports, Conex, Prague. 
Poulmedis, P.; Rondoyannis, G.; Mitsou, A., \& Tsarouchas, E. (1988). The influence of isokinetic muscle torque exerted in various speeds on soccer ball velocity. J ournal of Orthopaedics and Sports Physical Therapy, 10(3), 93-96.

Putnam, C. A. (1991). A segment interaction analysis of proximal to distal secuential segment motion patterns. Medicine and Science in Sports and Exercise, 23(1), 130144.

Rodano, R., \& Tavana, R. (1993). Three-dimensional analysis of instep kick in profesional soccer players. En T. Reilly, J. Clarys y A. Stibbe (Eds.), Science and Football (Vol. II, pp. 357-361). London: E \& FN Spon.

Shan, G., \& Westerhoff, P. (2005). Full-body kinematic characteristics of the maximal instep soccer kick by male soccer players and parameters related to kick quality. Sports Biomechanics, 4(1), 59-72.

Taïana, F.; Grehaigne, J., \& Cometti, G. (1993). The influence of maximal strength training of lower limbs of soccer players on their physical and kick performances. En T. Reilly, J. Clarys y A. Stibbe (Eds.), Science and Football (Vol. II, pp. 98-103). London: E \& FN Spon.

Trolle, M.; Aagaard, P.; Simonsen, J.; Bangsbo, J., \& Klaysen, K. (1993). Effects of strength training on kickin performance in soccer. En T. Reilly, J. Clarys y A. Stibbe (Eds.), Science and Football (Vol. II, pp. 95-98). London: E \& FN Spon.

Venturelli, M.; Trentin, F.; \& Bucci, M. (2007). Strength training for young soccer players. J ournal of Sports Sciences and Medicine, Suppl 10, 80-81.

Williams, M., \& Griffiths, I. W. (2002). A kinematic analysis of the prevalence of preimpact cues in the football penalty kick. Journal of Sports Sciences, 20(1), 74. 